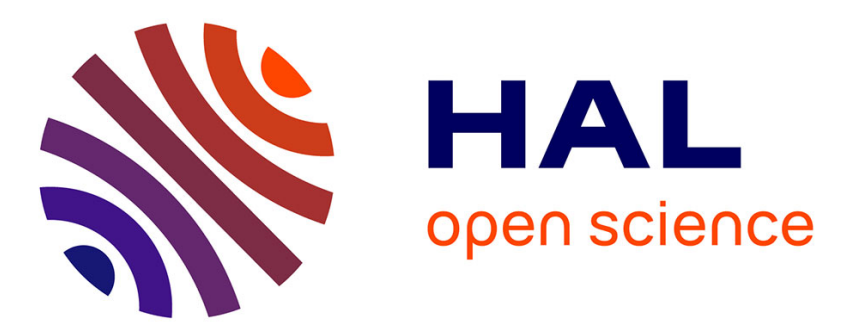

\title{
Elemental and isotopic fractionation of noble gases in gas and oil under reservoir conditions: Impact of thermodiffusion $\star$
}

\author{
Hai Hoang, Phuc Nguyen, Magali Pujol, Guillaume Galliero
}

\section{To cite this version:}

Hai Hoang, Phuc Nguyen, Magali Pujol, Guillaume Galliero. Elemental and isotopic fractionation of noble gases in gas and oil under reservoir conditions: Impact of thermodiffusion $\star$ European Physical Journal E: Soft matter and biological physics, 2019, 42 (5), 10.1140/epje/i2019-11823-x . hal-02749725

\section{HAL Id: hal-02749725 \\ https://hal.science/hal-02749725}

Submitted on 9 Jun 2021

HAL is a multi-disciplinary open access archive for the deposit and dissemination of scientific research documents, whether they are published or not. The documents may come from teaching and research institutions in France or abroad, or from public or private research centers.
L'archive ouverte pluridisciplinaire HAL, est destinée au dépôt et à la diffusion de documents scientifiques de niveau recherche, publiés ou non, émanant des établissements d'enseignement et de recherche français ou étrangers, des laboratoires publics ou privés. 


\title{
Elemental and Isotopic Fractionation of Noble Gases in Gas and Oil under reservoir conditions: Impact of Thermodiffusion
}

\author{
Hai Hoang ${ }^{1}$, Phuc Nguyen ${ }^{2}$, Magali Pujol ${ }^{3}$, Guillaume Galliero ${ }^{4 *}$ \\ ${ }^{1}$ Institute of Fundamental and Applied Sciences, Duy Tan University, 10C Tran Nhat Duat Street, \\ District 1, Ho Chi Minh City 700000, Viet Nam \\ ${ }^{2}$ Ho Chi Minh University of science, 227 Nguyen Van Cu, District 5, Ho Chi Minh City 7000, \\ Vietnam \\ ${ }^{3}$ TOTAL S.A., CSTJF, Avenue Larribau, Pau, 64018, France \\ ${ }^{4}$ Laboratoire des Fluides Complexes et leurs Réservoirs (UMR-5150 with CNRS, and TOTAL), \\ Université de Pau et des Pays de l'Adour, BP 1155, F-64013 Pau Cedex, France \\ *guillaume.galliero@univ-pau.fr
}

\begin{abstract}
Noble gases, and the way they fractionate, is a promising approach to better constrain origin, migration and initial state distributions of fluids in gas and oil reservoirs. Thermodiffusion, is one of the phenomena that may lead to isotope and elemental fractionation of noble gases. However, this effect, assumed to be small, has not been quantified, nor measured, in oil and gas under reservoir conditions. Thus, in this work, molecular dynamics simulations have been performed to compute the thermal diffusion factors of noble gases, in a dense gas (methane) and in an oil (n-hexane) under high pressures. Interestingly, it has been found that thermal diffusion factors, associated to both isotopic $\left({ }^{36} \mathrm{Ar},{ }^{40} \mathrm{Ar}\right)$ and elemental fractionations of noble gases $\left({ }^{4} \mathrm{He},{ }^{20} \mathrm{Ne},{ }^{40} \mathrm{Ar},{ }^{84} \mathrm{Kr}\right.$ and $\left.{ }^{131} \mathrm{Xe}\right)$ in gas and oil, could be expressed as linear functions of the reduced masses. Regarding the amplitude of the phenomena, it has been found that, in a stationary 1D oil or gas fluid column, thermodiffusion due to a typical geothermal gradient has an impact on noble gas isotopic and elemental fractionation which is of the same order of magnitude than gravity segregation, but opposite in sign. In addition, the relative impact of thermodiffusion on isotopic and elemental fractionations depends on the fluid type which is another interesting feature. Thus, these first numerical results on isotopic and elemental fractionation of noble gases by thermodiffusion in simple pure gas and oil emphasize their interest as natural tracers that could be used to improve the pre-exploitation description of oil and gas reservoirs.
\end{abstract}




\section{Introduction}

Noble gases are interesting and promising natural tracers allowing characterizing the origin, migration, and trapping of hydrocarbon reserves [1-3]. They are naturally present under traces in all natural fluids and can be measured accurately [1-3]. Interestingly, Noble gases are chemically and biologically inert under geological conditions and are so fractionated, i.e. partially separated, by physical processes only. Furthermore, they can be fractionated from the elemental (i.e. between the major isotopes of noble gases), and the isotopic (i.e. between isotopes of a given noble gas) points of view [1-3].

Existing physical processes which may lead to noble gas fractionation have been reviewed in literature [1-4]. They can be classified into two main families. The first one includes the mechanisms yielding fractionations at the stationary state, such as phase equilibrium or gravity segregation [1-3]. The second one is corresponding to the fractionation mechanisms associated to transient state, such as mass diffusion processes [1-4]. This work is a contribution to the former one.

The elemental and isotopic fractionations of noble gases in a gas and oil reservoir at the stationary state could be, in principle, quantitatively estimated by an ad hoc thermodynamic model [5-7], provided that corresponding physical processes are well defined and quantified. As a first approximation, the fluids are usually assumed to be subject to only the gravity field, which, for the noble gases, induces a relative enrichment of the heaviest isotopes and heaviest noble gases at the bottom of the fluid column [8-9]. However, vertical compositions of the fluids computed from this assumption may significantly differ from the actual ones [7, 10-12], i.e. the fractionations are incorrect. So, one has to introduce other external forces that may occur.

One of other external forces that may have a non-negligible impact on the elemental and isotropic fractionations of noble gases in a fluid column is due to a thermodiffusion induced by the geothermal gradient $[7,12]$. However, this phenomenon has not been paid much attention regarding elemental and isotopic fractionation of noble gas in geological fluids [1-3]. This is probably due to lack of data on the thermodiffusion (quantified by thermal diffusion factor) for the noble gases in geological fluids under reservoir conditions. This effect has often been assumed to be small as it could be deduced from the kinetic theory at low density gas conditions [13]. However, the thermal diffusion factor in simple fluids usually increases with density [14], and so may be significant in dense states such as those typical of reservoir conditions. Thus, one of the main objectives of this work is to provide a quantification of the elemental and isotopic fractionation of noble gases in gas and oil by the thermodiffusion under reservoirs conditions to check if it could be considered as a negligible phenomenon compared to the gravity segregation. 
Despite of non-negligible number of theoretical and empirical models proposed in literature, the thermal diffusion factor is difficult to predict in dense phase in particular when considering multicomponent fluids [15-17]. Furthermore, experiments under high pressures and on multicomponent mixtures, despite noticeable progresses [17], are still very difficult to achieve. During the last twenty years, molecular dynamics simulations have proved to be an alternative tool to estimate the thermal diffusion factor in simple fluids $[15,17]$. The accuracy of this tool is mainly controlled by description of potential energy between molecules, i.e. the force fields. With recent progresses in the molecular modelling, molecular simulations have shown to provide reasonable results for thermophysical properties, including the thermal diffusion factor, of various fluids including noble gases, natural gases, n-alkanes [18-19]. Thus, in this work, to quantify the isotopic $\left({ }^{36} \mathrm{Ar},{ }^{40} \mathrm{Ar}\right)$ and elemental fractionation $\left({ }^{4} \mathrm{He},{ }^{20} \mathrm{Ne},{ }^{40} \mathrm{Ar},{ }^{84} \mathrm{Kr}\right.$ and $\left.{ }^{131} \mathrm{Xe}\right)$ of noble gases by the thermodiffusion in gas and oil under high pressures, we have used molecular dynamics simulations combined with a recently developed force field.

Organization of the paper is as follows. In section II, thermodynamic model used to compute fractionation by gravity segregation and thermogravitation (gravity segregation + thermodiffusion) is presented. Details on molecular dynamics simulations are provided in section III. In section IV, results obtained from molecular simulations and the application of thermodynamic model are discussed. Finally, main results are summarized to form the conclusion in section V.

\section{Thermodynamic model of thermogravitation}

We consider here a one-dimension multicomponent oil/gas column, containing noble gases, with impermeable boundaries. If the gravity field effect is first considered, taken as constant, on the fluid column species distribution, the isothermal distribution of noble gases at equilibrium can be estimated by [8]:

$$
\left(\frac{d \mu_{\mathrm{NG}}}{d z}\right)_{T}=M_{N G} \times g
$$

where $\mu_{N G}$ and $M_{N G}$ are the chemical potential and the molecular weight of the considered noble gas, respectively, $z$ is the direction of the gravity field applied, $T$ is the temperature, and $g$ is the gravitational acceleration. As the noble gases are highly diluted in the oil and gas under reservoir conditions, their chemical potential in the mixture can be expressed as [20]:

$$
\mu_{\mathrm{NG}}=R T \ln x_{N G}+\mu_{0}
$$


where, $R$ is the gas constant, $x_{N G}$ is the molar fraction of noble gas, and $\mu_{0}$ is a function independent of $x_{N G}$. In addition, we can write that [20]:

$$
\left(\frac{d \mu_{N G}}{d p}\right)_{T, x}=v_{N G}
$$

where $v_{N G}$ is the partial molar volume of the considered noble gas and $P$ is the pressure. From Eqs. (2) and (3), the differential of the chemical potential of the considered noble gas in an isothermal mixture is so given as:

$$
d \mu_{\mathrm{NG}}=R T \frac{d x_{N G}}{x_{N G}}+v_{N G} d p
$$

In addition, the hydrostatic equilibrium of the fluid column imposes that:

$$
d p=\rho g d z
$$

where, $\rho$ is the density of mixture. Substituting Eqs. (4) and (5) into Eq. (1), we obtain:

$$
\frac{d x_{N G}}{x_{N G}}=\frac{g}{R T}\left(M_{N G}-\rho v_{N G}\right) d z
$$

When the vertical geothermal gradient, which is typically of $0.03 \mathrm{~K} / \mathrm{m}$, is taken into account in the modeling, a thermodiffusion term should be added to Eq. (6) which becomes:

$$
\frac{d x_{N G}}{x_{N G}}=\frac{g}{R T}\left(M_{N G}-\rho v_{N G}\right) d z-\frac{\alpha_{T, N G}}{T}\left(1-x_{N G}\right) d T
$$

where $\alpha_{T, N G}$ is the thermal diffusion factor of the considered noble gas in the mixture which is defined as usually by:

$$
\alpha_{T, N G}=-\frac{T}{x_{N G}\left(1-x_{N G}\right)} \times \frac{\nabla x_{N G}}{\nabla T}
$$

Once the vertical composition has been known at the stationary state from Eq. (6) (gravitational segregation) or Eq. (7) (thermogravitation), it is possible to quantify the fractionation $F$ between two noble gases, or two isotopes of a given noble gas, using:

$$
F\left[\frac{N G_{1}}{N G_{2}}\right]=\frac{\left(x_{N G_{1}} / x_{N G_{2}}\right)}{\left(x_{N G_{1}} / x_{N G_{2}}\right)_{\mathrm{Ref}}}
$$

where $(\ldots)_{\text {Ref }}$ is the value at a reference point.

\section{Fluid models and molecular simulations}

\subsection{Fluid models}


The Mie chain coarse-grained (MCCG) force field has been used to model the noble gases (Helium, Neon, Argon, Krypton and Xenon) and n-alkane molecules (methane and n-hexane), as it has shown a good capability to describe thermophysical properties of these species [21-23]. The MCCG model consists in a simple homo-nuclear chain composed of $N$ particles which are freely and tangentially bonded. The non-bonded interactions between particles are described by the Mie $\lambda-6$ potential:

$$
u_{M i e}\left(r_{i j}\right)=C \epsilon_{i j}\left[\left(\frac{\sigma_{i j}}{r_{i j}}\right)^{\lambda_{i j}}-\left(\frac{\sigma_{i j}}{r_{i j}}\right)^{6}\right]
$$

where $r_{i j}$ is the distance between the two particles, $\epsilon_{i j}$ is the potential well depth, $\sigma_{i j}$ is the collision diameter, $\lambda_{i j}$ is the exponent characterizing the repulsive interactions between the non-bonded particles and $C$ is the normalization factor defined as:

$$
C=\left(\frac{\lambda_{i j}}{\lambda_{i j}-6}\right)\left(\frac{\lambda_{i j}}{6}\right)^{6 /\left(\lambda_{i j}-6\right)}
$$

Two adjacent particles in a chain are connected by a bond with a fixed length of $\sigma_{i j}$. Hence, the MCCG force field requires four parameters $(N, \lambda, \epsilon, \sigma)$ to describe a compound.

To determine the four parameters of the MCCG force field, we have used a parameterization strategy proposed by Hoang et al. [23], except for Helium. This strategy is based on an extended corresponding states principle that requires the critical temperature, one saturated liquid density, the acentric factor and one saturated liquid reduced viscosity for each compound. This strategy could not be applied to Helium due to the quantum effect on the critical properties [24]. Hence, a LennardJones potential, which is corresponding to the MCCG force field with $N=1$ and $\lambda=12$, with the parameters proposed in literature has been used to model these compounds [25]. The values of the four parameters of the studied species are given in Tab. 1.

It is worth noting that the thermal diffusion factor is known to be very sensitive to the crossinteraction description [26-27]. In this work, we have employed the classical Lorentz-Berthelot combining rules to determine the cross-interaction parameters of $\sigma_{i j}$ and $\epsilon_{i j}$ using [28-29]:

$$
\begin{gathered}
\sigma_{i j}=\frac{\sigma_{i i}+\sigma_{j j}}{2} \\
\epsilon_{i j}=\sqrt{\epsilon_{i i} \times \epsilon_{j j}}
\end{gathered}
$$

And, the repulsion exponent of the cross-interactions, $\lambda_{i j}$, has been deduced from an arithmetic average as [30]: 


$$
\lambda_{i j}=\frac{\lambda_{i i}+\lambda_{j j}}{2}
$$

Hoang et al. have shown that the MCCG force field combined with these combining rules is able to reasonably predict the thermal diffusion factors for $\mathrm{Ar}-\mathrm{Kr}$ mixtures and n-pentane/n-decane mixtures at atmospheric pressures [23].

To evaluate the capability of this force field, with the chosen combining rules, to deal with mixtures of n-alkane with noble gases we have considered the Henry's constant of Xe in n-C6 defined as [31]:

$$
H_{N G}=\lim _{x_{N G}^{l i q} \rightarrow 0}\left[\frac{f_{N G}^{l i q}}{x_{N G}^{l i q}}\right]=\rho_{S}^{l i q} k_{B} T e^{\left(\mu_{N G, e x} / k_{B} T\right)}
$$

where, $f_{N G}^{l i q}$ and $x_{N G}^{l i q}$ are the fugacity and the mole fraction of Xe in the liquid n-C6 respectively, $\rho_{S}^{l i q}$ is the number density of the liquid n-C6, and $\mu_{N G, \text { ex }}$ is the excess chemical potential of Xe in the liquid n-C6. To determine $\rho_{S}^{\text {liq }}$, the Gibbs Ensemble Monte Carlo simulations have been carried out [32-33], whereas the Widom method has been employed to compute $\mu_{N G \text {,ex }}$ [34-35]. As for thermodiffusion, this quantity is very strongly controlled by the cross-interaction potential [20]. Results shown in Figure 1 indicate a good agreement between the Henry's constant from the experiment and from molecular simulations [36], confirming the good capability of the chosen force field to deal with the studied mixtures.

\subsection{Molecular simulations}

\section{Numerical details:}

In all molecular simulations, a cutoff radius equal to $3.5 \sigma$ has been used and long range corrections have been included [37]. To constrain the bond length, we have employed the classical RATTLE algorithm [38]. When required, temperature and pressure have been controlled using a Berendsen algorithm [39].

Simulation boxes composed of between 1000 and 3000 molecules were used to compute the properties. After equilibration or after reaching the steady state, samplings have been carried out during about $5 \times 10^{7}$ time steps. To improve the statistical uncertainties, self-diffusions and thermal diffusion factors presented in the following correspond to averages on between 5 to 10 independent runs for each configuration, and the error bars are corresponding to the standard deviations [18]. 


\section{Transport Properties computation:}

To estimate the thermal diffusion factor of noble gases in the studied fluids, we have employed a boundary driven non-equilibrium molecular dynamics (NEMD) scheme proposed by Müller-Plathe [40]. In this scheme, a simulation box is divided into $N_{S}$ slabs (24 in this work) along the direction of the thermal gradient, and the thermal gradient is generated by exchanging the kinetic energies of molecules in the central part of simulation box, $N_{S} / 2$ and $N_{S} / 2+1$, with the edge slabs, 1 and $N_{S}$. At the stationary state, the local composition and temperature are computed, and the thermal diffusion factor is simply deduced from Eq. (8). According to this formulation, a positive (negative) value of $\alpha_{T}$ corresponds to a relative migration of the noble gas towards the cold (hot) region.

In addition, the thermal diffusion coefficients of the noble gases have been computed from the thermal diffusion factors, the mass diffusion coefficients $D_{N G}$ and the temperature using [16]:

$$
D_{T, N G}=D_{N G} \frac{\alpha_{T, N G}}{T}
$$

In which, the mass diffusion coefficients are simply equal to their corresponding self-diffusion coefficients, $D_{S, N G}$, as the noble gases are highly diluted for the studied systems [41]. To compute the self-diffusion coefficients of noble gases, we have used a usual Einstein relation during equilibrium molecular dynamics (EMD) simulations as [42]:

$$
D_{S, N G}=\lim _{t \rightarrow \infty} \frac{1}{6 t}\left\langle\left[\mathbf{r}_{i}(t)-\mathbf{r}_{i}(0)\right]^{2}\right\rangle
$$

where, $t$ is the time, $\langle\ldots\rangle$ is the average over molecules and $\mathbf{r}_{i}(t)$ is the position of molecule $i$ at the time $t$.

One of the main challenges of the proposed strategy to compute thermal diffusion factors of noble gases in methane and in n-hexane under reservoir conditions is that noble gases are highly diluted (with typical mole fraction of the order of $10^{-5} \div 10^{-3}$ [43]). This may lead to simulation results with a poor statistics and so to very large error bars [44-45]. So, to improve the statistics, we have computed the dependence of the density and the thermal diffusion factor of the ${ }^{40} \mathrm{Ar}+\mathrm{nC} 6$ mixture on the mole fraction of ${ }^{40} \mathrm{Ar}$ at $T=323 \mathrm{~K}$ and $P=10 \mathrm{MPa}$. Figure 2 indicates that simulation systems containing about $1.0 \%$ mole fraction of ${ }^{40} \mathrm{Ar}$ are a good approximation of highly diluted systems such as those found in natural reservoirs. Hence, the number of noble gas molecules used in this work corresponds to a mole fraction equal to $1.0 \%$ in all studied systems.

\section{Results and Discussions}




\subsection{Thermal diffusion factor and thermal diffusion coefficient}

Molecular dynamics simulations have been performed at thermodynamic conditions corresponding to a temperature of $323 \mathrm{~K}$ and a pressure of $100 \mathrm{MPa}$ for methane (dense gas) and of $10 \mathrm{MPa}$ for n-hexane (oil). Such pressure range from 10 to $100 \mathrm{MPa}$ covers what usually found in oil and gas reservoirs. However, it should be noticed that the gas pressure used in this work is relatively high compared to what usually found in natural reservoirs. This choice was guided by the idea of mimicking a more complex dense gas mixture with the use of a simple gas composed of methane under high pressures.

\subsubsection{Thermal diffusion factor}

\section{A. Noble Gases}

Figure 3 shows the thermal diffusion factor for the major isotopes of noble gases in the dense gas and in the oil obtained from NEMD simulations. As expected, thermal diffusion factors increase with the weight of the of the noble gas both in the dense gas and in the oil with values typical of those encountered for simple fluids, i.e. values of thermal diffusion factors of the order of one [14, 46], which is not negligible. Furthermore, in the dense gas all noble gases bigger than Ne tends to migrate to the cold area $\left(\alpha_{T, N G}>0\right)$, whereas only those bigger than Ar tends to migrate to the cold area in the oil. Thus, a noble gas that has a molecular mass greater than that of its solvent, tends to migrate, relatively to the solvent, to the cold region. In addition, except for Helium in dense gas, it seems that thermal diffusion factors of the noble gases are linearly related to the reduced mass $\delta M$ defined by:

$$
\delta M=\left(M_{N G}-M_{S}\right) /\left(M_{N G}+M_{S}\right)
$$

where $M_{S}$ is the mass of the solvent (here Methane or n-Hexane).

To understand this linear relationship, it should be first recalled that the thermal diffusion factor of a binary mixture is usually decomposed in two contributions as [17, 47]:

$$
\alpha_{T}=\alpha_{T, I s o}+\alpha_{T, \text { Chem }}
$$

where $\alpha_{\mathrm{T}, \mathrm{Iso}}$ is the isotopic contribution, i.e. a mass effect, and $\alpha_{\mathrm{T}, \mathrm{Chem}}$ is the chemical contribution, i.e. a contribution from molecular interactions. The isotopic contribution is known to be well described by:

$$
\alpha_{T, I s o}=\alpha_{T, 0}^{M} \times \delta M
$$


where, $\alpha_{T, 0}^{M}$ is a coefficient independent on the molecular mass. Thus, a linear relationship between $\alpha_{\mathrm{T}}$ and $\delta M$ for noble gases in the dense gas and the oil implies that the parameters of the interaction potential, which contributes to $\alpha_{T, \text { Chem, }}$ possess a "peculiar" relationship with the molecular mass.

To support this idea, it is interesting to note that, when considering noble gases (except Helium for quantum reasons) and n-alkanes, $V_{c}^{-2 / 3}$ scales linearly with $M^{-1 / 2}$, see Fig. 4 , where $V_{c}$ is the critical volume [48]. This means that, for these species, the reciprocal of the square root of molecular mass $M^{-1 / 2}$ scales with the reciprocal of square of molecular size $r^{-2}$. For mixtures composed of species possessing such a relation between mass and size, the kinetic theory [13] leads to a thermal diffusion factor in gaseous states exhibiting a linear variation with the reduced mass. In addition, in dense states, Galliero et al. [14] and then De Mezquia et al. [49] have found that the thermal diffusion factors in binary liquid mixture of $n$-alkane can be quantitatively defined as a linear function of the reduced mass.

\section{B. Isotopes of Ar}

Thermal diffusion factors for "isotopes" of Argon, with isotopic mass going from $10 \mathrm{~g} / \mathrm{mol}$ to $90 \mathrm{~g} / \mathrm{mol}$, have been computed in the dense gas and in the oil. By using such unrealistic isotopes, it is possible to amplify the differences so as to better quantify them from the simulation point of view. Results are displayed in Fig 5. As expected, the heavier isotopes migrate more to the cold region than the lighter isotopes [13-14] in both the dense gas and in the oil.

It is interesting to notice that the thermal diffusion factor has a linear relationship with the reduced mass for the isotopes of Argon, see Fig. 5. This can be easily understood as the chemical contribution to thermal diffusion is constant, see Eqs. (17) and (18). Regarding the value of the slopes of the linear relationship, i.e. $\alpha_{T, 0}^{M}$, they have been found to be equal to $2.5 \pm 0.4$ and $4.3 \pm 0.9$ in the dense gas and in the oil, respectively. These values are consistent, especially in the dense gas state, with what could be deduced from the correlation developed by Galliero et al. [14] to deal with isotopic mixtures. More precisely this correlation yields $\alpha_{T, 0}^{M}=2.53$ for the dense gas and $\alpha_{T, 0}^{M}=3.14$ for the oil. Such a results means, that, in a first approximation, the correlation developed in Galliero et al. [14] seems to be adequate to deal with such systems.

\subsubsection{Thermal diffusion coefficient}

\section{A. Noble Gases}


Figure 6 shows thermal diffusion coefficients, $D_{T}$, of the major isotopes of noble gases in the dense gas and in the oil. Similarly what obtained on thermal diffusion factor, $D_{T}$ increases with the noble gas weight. Interestingly, it has been found that a linear relationship can be observed when $D_{T}$ is plotted against the reciprocal of the square root of the molecular mass $M_{N G}^{-0.5}$ of the considered noble gas. This is probably related to the fact that mass diffusion $D_{N G}$ is known to scale well with $M_{N G}^{-0.5}$ when dealing with noble gases diffusion in dense fluids (water) [50].

\section{B. Isotopes of $\mathrm{Ar}$}

Thermal diffusion coefficients for unrealistic isotopes of Argon in the dense gas and in the oil have been computed and are displayed in Fig. 7. Results indicate that $D_{T}$ increases with the isotopic mass, and linearly scales with the reduced mass as found for thermal diffusion factor $\alpha_{T}$. This is probably partially due to the fact the mass diffusion of isotopes does not change strongly with the mass when dealing with diluted solute in a dense solvent [50].

\subsection{Elemental and isotopic fractionation}

In this part, Eqs. (6) and (7) have been used to quantify the fractionation of noble gases in the dense gas $(\mathrm{T}=323 \mathrm{~K}, \mathrm{P}=100 \mathrm{MPa})$ and in the oil $(\mathrm{T}=323 \mathrm{~K}$ and $\mathrm{P}=10 \mathrm{MPa})$ due to gravitational segregation and thermogravitation at the stationary state. The models have been applied to a 1D fluid column of $200 \mathrm{~m}$ height with constant thermophysical properties, using the values computed from molecular simulations at the reference point. Regarding the partial molar volumes $v_{N G}$, they have been obtained using the Kirkwood-Buff theory [51] combined with the method proposed by Milzetti et al. [52].

\subsubsection{Elemental Fractionation}

Figures 8 and 9 show the isothermal distribution of the elemental fractionations between noble gases and ${ }^{40} \mathrm{Ar}$ along a fluid column from the reference position $z_{0}$ to $z_{0}-200 \mathrm{~m}$ due to the gravity field only in the dense gas and oil, respectively. As expected from the choice in the modeling (constant thermophysical properties), the elemental fractionation $\mathrm{F}\left[\mathrm{NG} /{ }^{40} \mathrm{Ar}\right]$, computed using eq. (9), linearly varies with the deep of fluid column $\left(z_{0}-z\right)$. More interesting is that the noble gases larger than ${ }^{40} \mathrm{Ar}$ are more enriched at the bottom of the fluid column than ${ }^{40} \mathrm{Ar}$, whereas it is opposite for the other noble gases. It is worth noticing that the elemental fractionations in both dense gas and in oil are rather similar. This can be explained by the fact that the partial molar volume varies slightly 
with the type of noble gas compared to the molecular mass, which leads to $\left|\rho\left(v_{N G}-v_{A r}\right)\right| \ll$ $\left|M_{N G}-M_{A r}\right|$. So, F[NG/Ar] is weakly dependent on the solvent (e.g. methane and n-hexane), see Eqs. (6) and (9).

When a geothermal gradient is applied to the dense gas and the oil, the elemental fractionations are induced by coupling between gravity segregation and thermodiffusion (thermogravitation), see Eq. (7). Results shown in Figs. 8 and 9 indicate that the smaller noble gases, i.e. ${ }^{4} \mathrm{He}$ and ${ }^{20} \mathrm{Ne}$, migrates more toward the bottom than ${ }^{40} \mathrm{Ar}$, which is opposite to what obtained when only the gravity field is considered. In addition, elemental fractionation of the larger noble gases, i.e. ${ }^{84} \mathrm{Kr}$ and ${ }^{131} \mathrm{Xe}$, exhibits the same trend than when only the gravity is taken into account but in a reduced manner. It is worth noticing that these behaviors are similar in both the gas and the oil phases.

These observations imply that the thermodiffusion has a crucial impact, sometimes even larger than gravity segregation, on the stationary state elemental fractionation of noble gases in the gas and oil reservoirs.

\subsubsection{Isotopic Fractionation}

Regarding the isotopic fractionation, we have studied the fractionation between ${ }^{36} \mathrm{Ar}$ and ${ }^{40} \mathrm{Ar}$ in the dense gas and in the oil. Figure 10 displays the distributions of this fractionation when only gravity field is applied. It is expectedly found that the isotropic fractionation $\mathrm{F}\left[{ }^{36} \mathrm{Ar} /{ }^{40} \mathrm{Ar}\right]$ linearly varies with the deep of fluid column $\left(z_{0}-\mathrm{z}\right)$, and the light isotope is less enriched at the bottom of fluid column than the heavy isotope. It should be noticed that $\mathrm{F}\left[{ }^{36} \mathrm{Ar} /{ }^{40} \mathrm{Ar}\right]$ due to the gravity field is similar in dense gas and in oil. This is simply due to the fact that the partial molar volumes of the two isotopes are the same as previously discussed for the elemental fractionation.

To investigate the fractionation between ${ }^{36} \mathrm{Ar}$ and ${ }^{40} \mathrm{Ar}$ when adding the geothermal gradient to the gravity field, we have used thermal diffusion factors of ${ }^{36} \mathrm{Ar}$ and ${ }^{40} \mathrm{Ar}$ deduced from a linear fitting on the simulation results obtained for Ar masses ranging from $10 \mathrm{~g} / \mathrm{mol}$ to $90 \mathrm{~g} / \mathrm{mol}$, see Fig. 5. The distribution of this fractionation is shown in Fig. 10. In the dense gas, the gravity segregation still dominates the fractionation but in the oil the effect of thermodiffusion is larger than that of the gravity field which leads to a reversed gradient compared to the gravitational case alone. It should be emphasized that contrary to what was obtained on the elemental fractionation, isotopic fractionations in the dense gas and in the oil are noticeably different when the thermodiffusion is taken into account. 


\section{Conclusions}

In this work, we have studied the effect of thermodiffusion on isotopic $\left({ }^{36} \mathrm{Ar},{ }^{40} \mathrm{Ar}\right)$ and elemental fractionations of noble gases $\left({ }^{4} \mathrm{He},{ }^{20} \mathrm{Ne},{ }^{40} \mathrm{Ar},{ }^{84} \mathrm{Kr}\right.$ and $\left.{ }^{131} \mathrm{Xe}\right)$ in dense gas (methane) and in oil (n-hexane) under reservoir conditions. To do so, we have used non-equilibrium molecular dynamics simulations to compute thermal diffusion factor and thermal diffusion coefficients of noble gases in both gas and oil. The Mie Chain Coarse Grained force field has been employed to model noble gases and n-alkanes molecules. Then, using a classical thermodynamic model and the properties computed from simulations, isotopic and elemental fractionations by gravity segregation and thermogravitation (gravity segregation + thermodiffusion) have been evaluated.

Interestingly, it has been found that thermal diffusion factors, associated to isotopic and elemental fractionations of noble gases in the dense gas and in the oil, could be simply expressed as linear functions of the reduced mass. Such a behavior was expected for the former, and for the latter this behavior could be explained by the fact that for the noble gases there is a linear relationship between the reciprocal of square root of molecular mass and the reciprocal of square of molecular size. In addition, it has been noticed that thermal diffusion coefficients exhibit a linear relationship with the reciprocal of square root of the molecular mass for the major isotopes of noble gases and with the reduced mass for isotopes of Argon. These behaviors could be attributed to behavior of the mass diffusion coefficient which was found to be linearly dependent on the reciprocal of square root of molecular mass for the major isotopes, whereas it is weakly dependent on the molecular mass for the isotopes of Argon.

In a second step, these results on thermodiffusion of noble gases have been used to compare the isotopic and elemental fractionations due to gravity segregation alone and due to thermogravitation using a typical geothermal gradient in a 1D fluid column. For the elemental fractionation, results have shown that the larger noble gases are more enriched at the bottom of the fluid column when only gravity field is present, with a similar amplitude in both the dense gas and in the oil. In the thermogravitation case, the relative noble gases distribution is drastically changed. More precisely, the distribution of the smaller noble gases, i.e. ${ }^{4} \mathrm{He}$ and ${ }^{20} \mathrm{Ne}$, is reversed compared to gravity segregation alone, whereas the magnitude of the fractionation of the larger noble gases, i.e.

${ }^{84} \mathrm{Kr}$ and ${ }^{131} \mathrm{Xe}$, is noticeably diminished. These behaviors have been found similar in both the dense gas and in the oil. Regarding isotopic fractionation between ${ }^{36} \mathrm{Ar}$ and ${ }^{40} \mathrm{Ar}$, it has been found that ${ }^{40} \mathrm{Ar}$ is enriched at the bottom of fluid column compared to ${ }^{36} \mathrm{Ar}$ if gravity segregation only is considered. When taking into account the additional contribution of thermodiffusion, this behavior is reversed in 
the oil, and noticeably diminished in the dense gas. Thus, it appears that the type of reservoir fluid has noticeable effect on the isotopic fractionation, but only a weak one on the elemental fractionation.

Thus, even if this work is only preliminary and deals only with pure gas and pure oil, these results emphasize the need of taking into account thermodiffusion when modeling noble gas isotopic and elemental fractionations in a stationary 1D oil or gas fluid column. In addition, because of the differences in the relative impact of thermodiffusion on isotopic and elemental fractionation of noble gases, these natural tracers are probably good candidates to confirm or infirm the stationary state, and probably the stability, of a fluid column.

\section{Acknowledgements:}

We gratefully acknowledge TOTAL S.A. for the post-doctoral grant awarded to one of us (HH) and for letting us publish these results. We also thank the Pau University and the MCIA for providing computational facilities.

\section{Author contribution Statement}

H.H. performed the simulations and P.N. contributed to the theory. G.G. proposed the approach and contributed to the developments. M.P. supervised and funded the project. H.H. took the lead in writing the manuscript with inputs from G.G and M.P. 


\section{References}

[1] M. Ozima and F. Podosek, Noble Gas Geochemistry, Cambridge Univ. Press. (2002)

[2] C. J. Ballentine, R. Burgess, B. Marty, Rev. Mineral. Geochem. 47, 539 (2002)

[3] P. Burnard, The Noble Gases as Geochemical Tracers, Springer (2013)

[4] B. Marty, Geochem. J. 18, 157 (1984)

[5] J. W. Gibbs, Collected Works - Vol. 1 - Thermodynamics, Yale University Press, New Haven (1957)

[6] G. Galliero and F. Montel, Phys. Rev. E 78, 041203 (2008)

[7] G. Galliero, H. Bataller, F. Croccolo, R. Vermorel, P. A. Artola, B. Rousseau, V. Vesovic, M. Bou-Ali, J. M. O. de Zarate, S. Xu, K. Zhang, F. Montel, Microgravity Sci. Technol. 28, 79 (2016).

[8] B. H. Sage, W. N. Lacey, Trans. AIME 132, 120 (1939)

[9] L. Høier, C. H. Whitson, SPE Reserv. Eval. Eng. 4, 525 (2001)

[10] T. Holt, E. Lindeberg, K. S. Ratkje, SPE Paper, 11761 (1983)

[11] C. H. Whitson, P. Belery, SPE Paper, 28000 (1994)

[12] F. Montel, J. Bickert, A. Lagisquet, G. Galliero, J. Pet. Sci. Eng. 58, 391 (2007)

[13] S. Chapman and T. G. Cowling, The Mathematical Theory of Non-Uniform Gases, Cambridge University Press, Cambridge, (1981).

[14] G. Galliero, M. Bugel, B. Duguay and F. Montel, J. Non-Equilib. Thermodyn. 32, 251 (2007)

[15] S. Wiegand, J. Phys.: Condens. Matter 16, R357 (2004)

[16] S. Srinivasan and M. Z. Saghir, Thermodiffusion in Multicomponent Mixtures: Thermodynamic, Algebraic, and Neuro-Computing Models, Springer Science \& Business Media, (2012)

[17] W. Köhler and K. I. Morozov, J. Non-Equilib. Thermodyn. 41, 151 (2016)

[18] P. Ungerer, B. Tavitian, A. Boutin, Applications of Molecular Simulation in the Oil and Gas industry, Technip (2005).

[19] M. Zhang and F. Müller-Plathe, J. Chem. Phys. 125, 124903 (2006) 
[20] M. J. Assael, J. M. P. Trusler and T. F. Tsolakis, Thermophysical Properties of Fluids. An Introduction to their Prediction, Imperial College Press (1996)

[21] A. Mejia, C. Herdes and E. A. Müller, Ind. Eng. Chem. Res. 53, 4131 (2014).

[22] E. A. Müller and G. Jackson, Annu. Rev. Chem. Biomol. Eng. 5, 405 (2014).

[23] H. Hoang, S. Delage-Santacreu, G. Galliero, Ind. Eng. Chem. Res. 56, 9213 (2017)

[24] R. D. Gunn, P. L. Chueh, J. M. Prausnitz, AIChE J. 12, 937 (1966)

[25] J. O. Hirschfelder, C.F. Curtiss, R.B. Bird, Molecular Theory of Gases and Liquids, Wiley, New York, (1954)

[26] P. A. Artola and B. Rousseau, Phys. Rev. Lett. 98, 125901 (2007)

[27] G. Galliero, S. Srinivasan, M. Z. Saghir, High Temp-High Press 38, 315 (2008)

[28] T. Schnabel, J. Vrabec, H. Hasse, J. Mol. Liq. 135,170 (2007)

[29] A. J. Haslam , A. Galindo, G. Jackson, Fluid Phase Equilib. 266, 105 (2008)

[30] J. R. Mick, M. S. Barhaghi, B. Jackman, K. Rushaidat, L. Schwiebert, and J. J. Potoff, J. Chem. Phys. 143, 114504 (2015)

[31] K. S. Shing, K. E. Gubbins, K. Lucas, Mol. Phys. 65, 1235 (1988)

[32] A. Z. Panagiotopoulos, Mol. Phys. 61, 813 (1987).

[33] A. Z. Panagiotopoulos, N. Quirke, M. Stapleton and D. J. Tildesley, Mol. Phys. 63, 527 (1988).

[34] B. Widom, J. Chem. Phys. 39, 2808 (1963)

[35] B. Widom, J. Phys. Chem. 86, 869 (1982)

[36] R. P. M. F. Bonifácio, M. F. C. Gomes, E. J. M. Filipe, Fluid Phase Equilib. 193, 41 (2002)

[37] M. P. Allen and D. J. Tildesley, Computer simulations of Liquids, Oxford University Press, New York, (1987).

[38] H. C. Andersen, J. Comput. Phys. 52, 24 (1983). 
[39] H. J. C. Berendsen, J. P. M. Postma, W. F. van Gunsteren, A. Dinola and J. R. Haak, J. Chem. Phys. 81, 3684 (1984).

[40] F. Müller-Plathe, D. Reith, Comput. Theor. Polymer Sci. 9, 203 (1999).

[41] L. S. Darken, Diffusion, Trans. AIME 1975, 184 (1948)

[42] J. M. Haile, Molecular Dynamics Simulation: Elementary Methods, John Wiley \& Sons, Inc. New York (1992)

[43] J. J. Ross, A literature survey of noble gas solubility measurements in formation brines to interpret tracer experiments, Bachelor's thesis, Ohio State University (2018)

[44] G. Galliero and S. Volz, J. Chem. Phys. 128, 064505 (2008).

[45] M. Yang and M. Ripoll, J. Phys.: Condens. Matter 24, 195101 (2012)

[46] G. Galliero, B. Duguay, J. P. Caltagirone and F. Montel, Fluid Phase Equilib. 208, 171 (2003)

[47] C. Debuschewitz and W. Köhler, Phys. Rev. Lett. 87, 055901 (2001)

[48] E. W. Lemmon, M. L. Huber, M. O. McLinden, Reference Fluid Thermodynamic and Transport Properties, NIST Standard Reference Database 23, REFPROP Version 8.0 (2007)

[49] D. A. de Mezquia, M. M. Bou-Ali, J. A. Madariaga, and C. Santamaría, J. Chem. Phys. 140, $084503(2014)$

[50] I. C. Bourg, G. Sposito, Geochim. Cosmochim. Acta 72, 2237 (2008)

[51] J. G. Kirkwood and F. P. Buff, J. Chem. Phys. 19, 774, (1951).

[52] J. Milzetti, D. Nayar, N. F. A. van der Vegt, J. Phys. Chem. B 122, 5515 (2018) 
Table 1: Parameters of the MCCG force field used to model the noble gases in normal alkanes.

\begin{tabular}{|l|c|c|c|c|}
\hline Compound & $\mathrm{N}$ & $\lambda$ & $\varepsilon(\mathrm{J} / \mathrm{mol})$ & $\sigma(\AA)$ \\
\hline $\mathrm{He}$ & 1 & 12.00 & 90.628 & 2.640 \\
\hline $\mathrm{Ne}$ & 1 & 12.51 & 290.28 & 2.813 \\
\hline $\mathrm{Ar}$ & 1 & 13.93 & 1044.1 & 3.407 \\
\hline $\mathrm{Kr}$ & 1 & 13.43 & 1423.0 & 3.634 \\
\hline $\mathrm{Xe}$ & 1 & 14.22 & 2029.8 & 3.962 \\
\hline $\mathrm{CH}_{4}$ & 3 & 11.06 & 1150.2 & 3.707 \\
\hline $\mathrm{nC}_{6}$ & 1 & 13.38 & 2227.3 & 3.862 \\
\hline
\end{tabular}


Figure 1: Henry's law coefficients of Xenon in Hexane obtained from experiments [36] and from molecular simulations using MCCG force field.

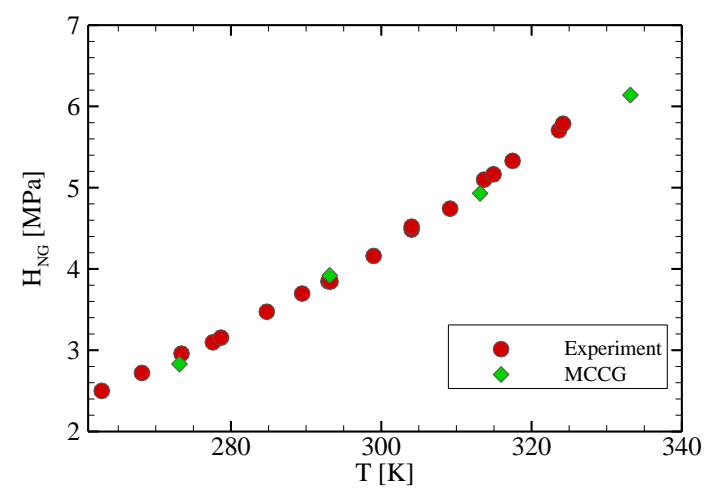

Figure 2: Dependence of thermophysical properties on the molar fraction of noble gas for the binary mixture of Ar+nC6at $T=323 \mathrm{~K}$ and $P=10 \mathrm{MPa}$. (a) Density. (b) Thermal diffusion factor.
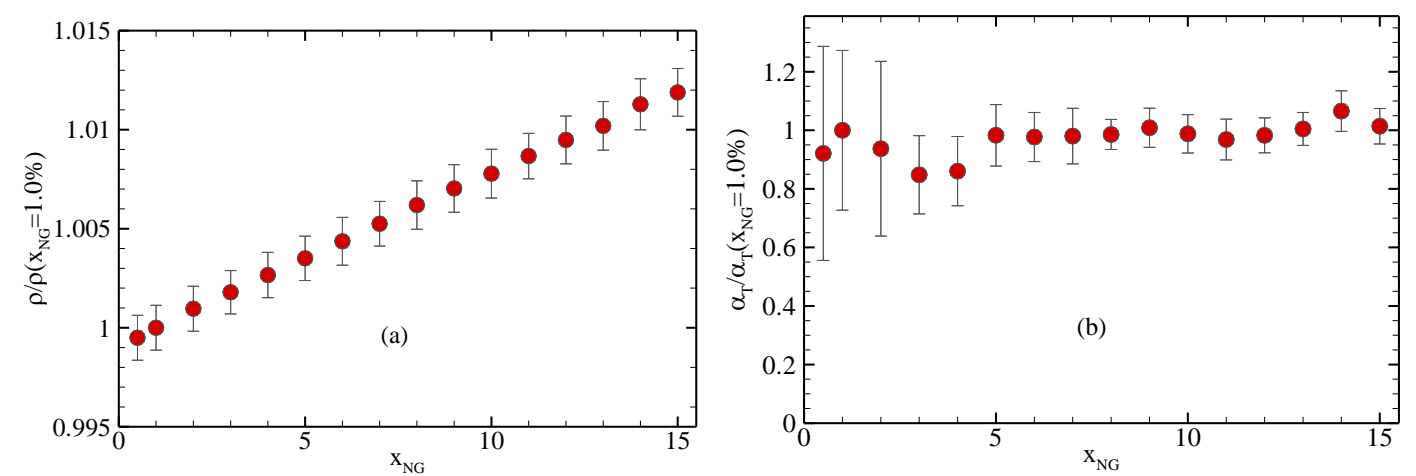

Figure 3: Reduced mass dependence of thermal diffusion factor of noble gases in dense gas (a) and in oil (b). Symbols correspond to the simulation results. Curves are linear fitting to the simulation results and serve a guide for the eye.
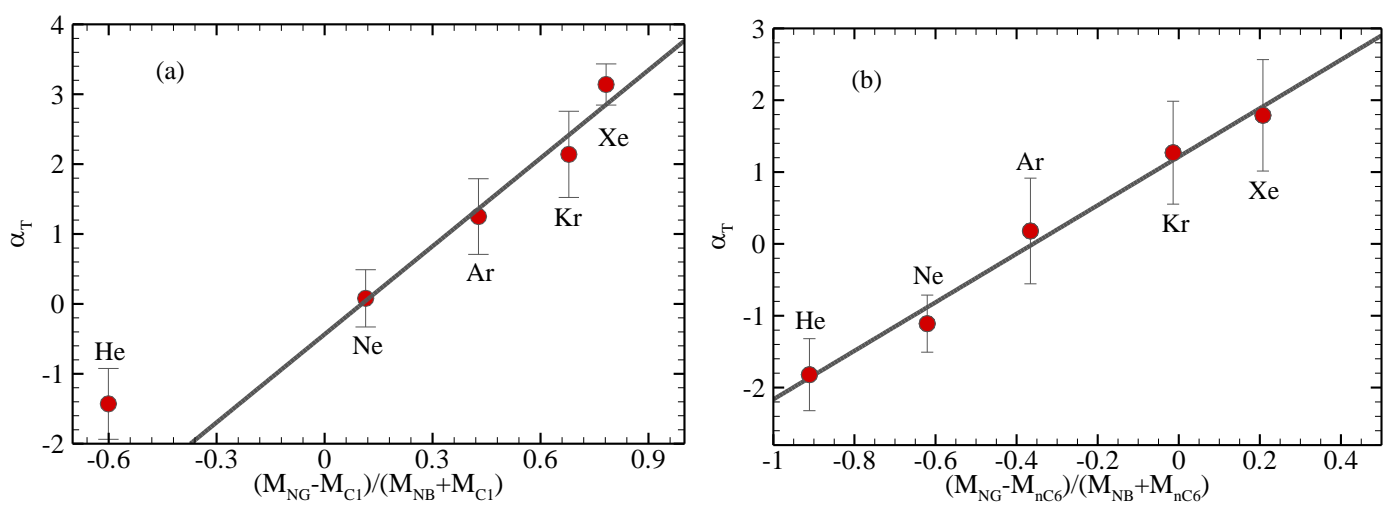
Figure 4: Relationship between $M^{-1 / 2}$ and $V_{C}^{-2 / 3}$ in n-alkanes and in noble gases [48].

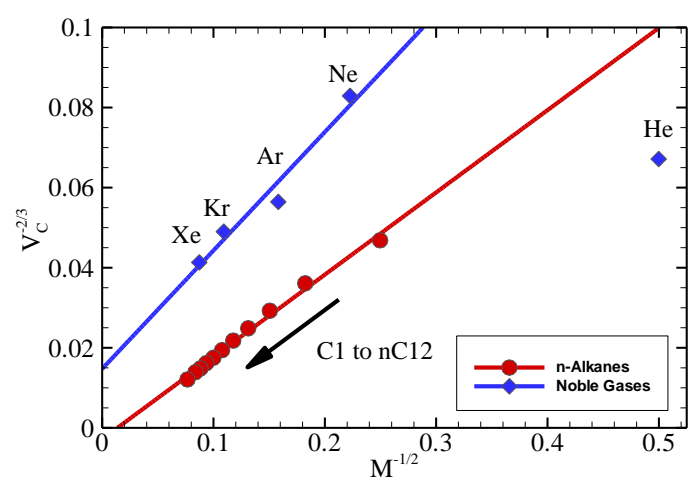

Figure 5: Reduced mass dependence of thermal diffusion factor of "isotopes" of Argon with isotopic masses ranging from $10 \mathrm{~g} / \mathrm{mol}$ to $90 \mathrm{~g} / \mathrm{mol}$ in the dense gas (a) and in the oil (b). Green diamonds correspond to ${ }^{40} \mathrm{Ar}$.
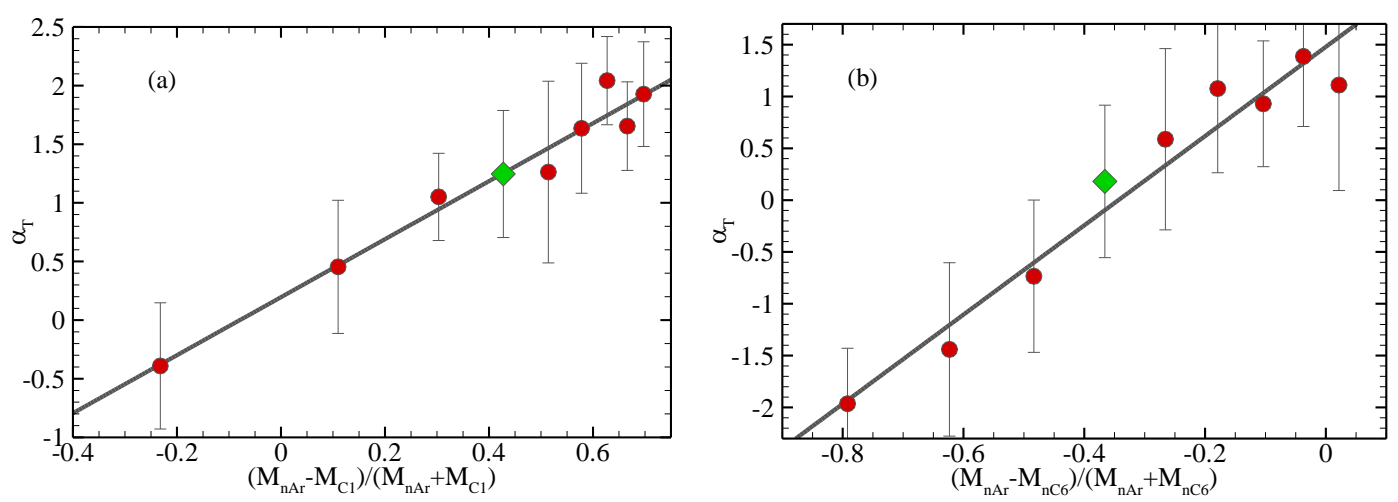
Figure 6: Thermal diffusion coefficients versus reciprocal of square root of molecular mass of noble gases. (a) In dense gas. (b) In oil. The legend is the same as that in Fig. 3.
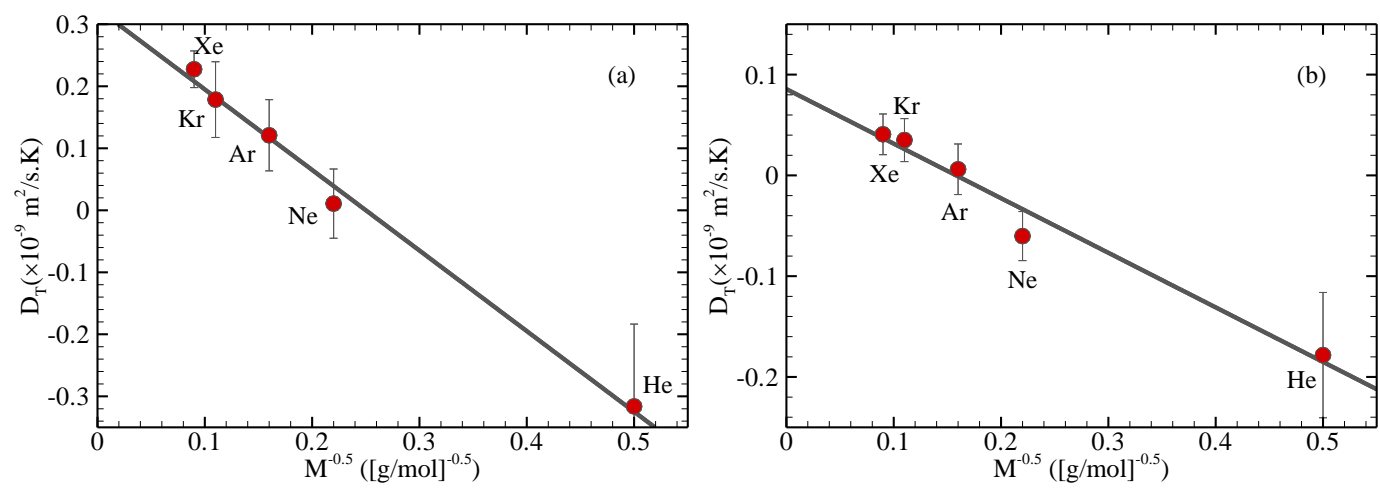

Figure 7: Thermal diffusion coefficients versus reduced mass of "isotopes" of Argon. (a) In dense gas. (b) In oil. The legend is the same as that in Fig. 5.
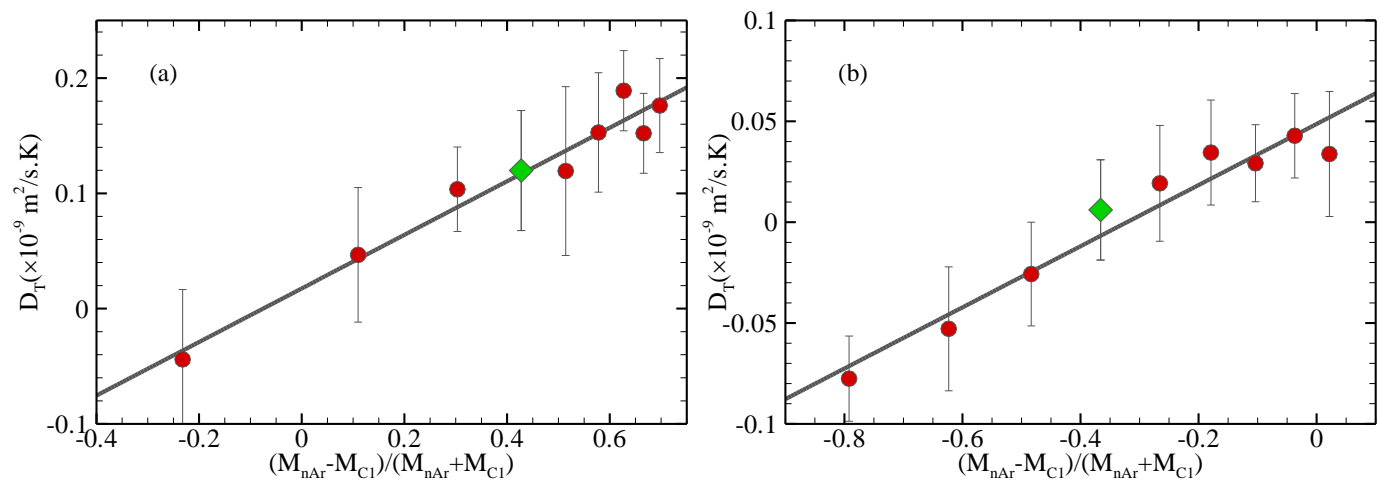
Figure 8: Vertical distribution of elemental fractionation due to gravity segregation (red) and due to thermogravitation (green) in dense gas.
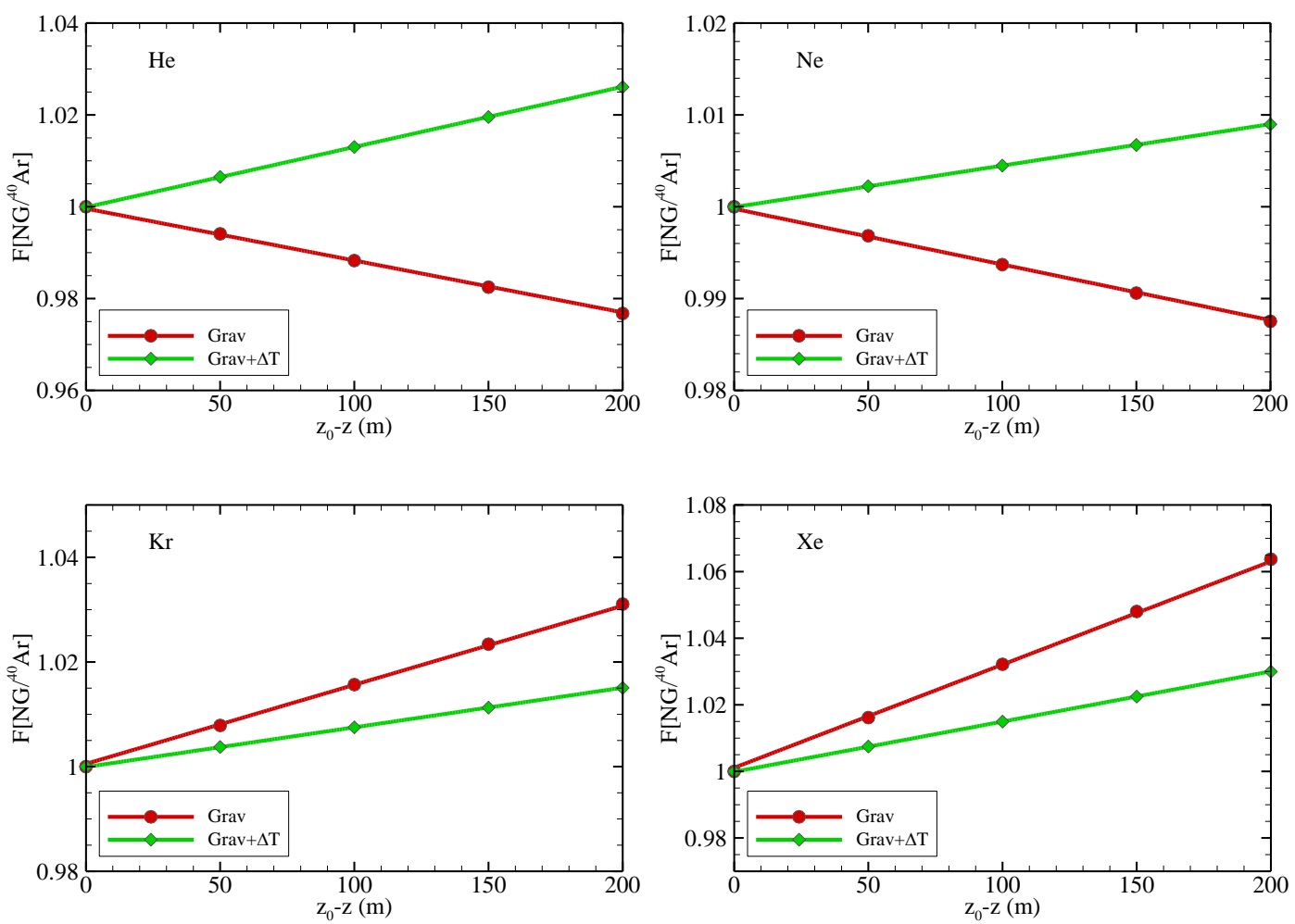
Figure 9: Vertical distribution of elemental fractionation due to gravity segregation (red) and due to thermogravitation (green) in oil.
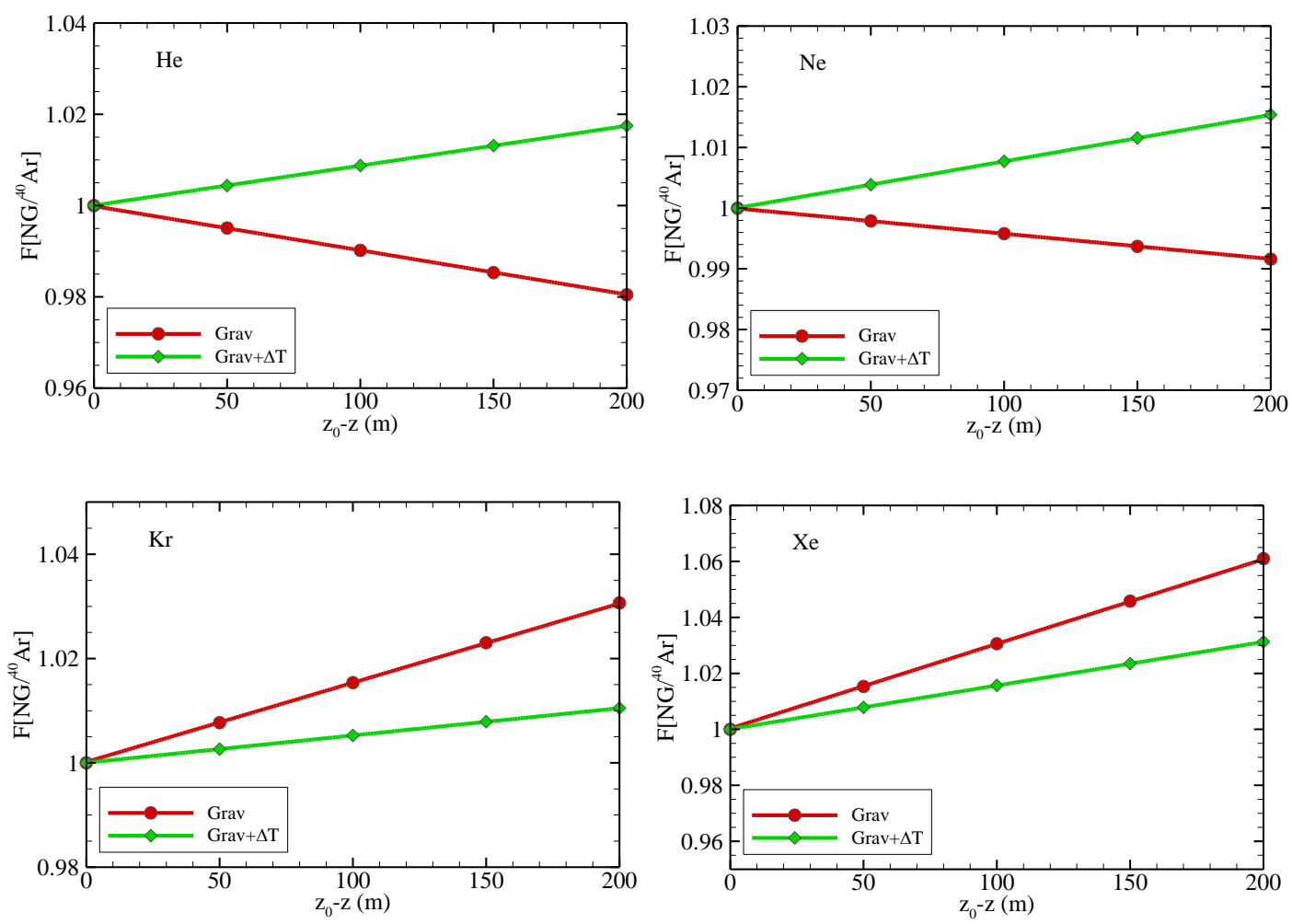
Figure 10: Vertical distribution of the isotopic fractionation between ${ }^{36} \mathrm{Ar}$ and ${ }^{40} \mathrm{Ar}$ due to gravity segregation (red) and due to thermogravitation (green). (a) In dense gas. (b) In oil.
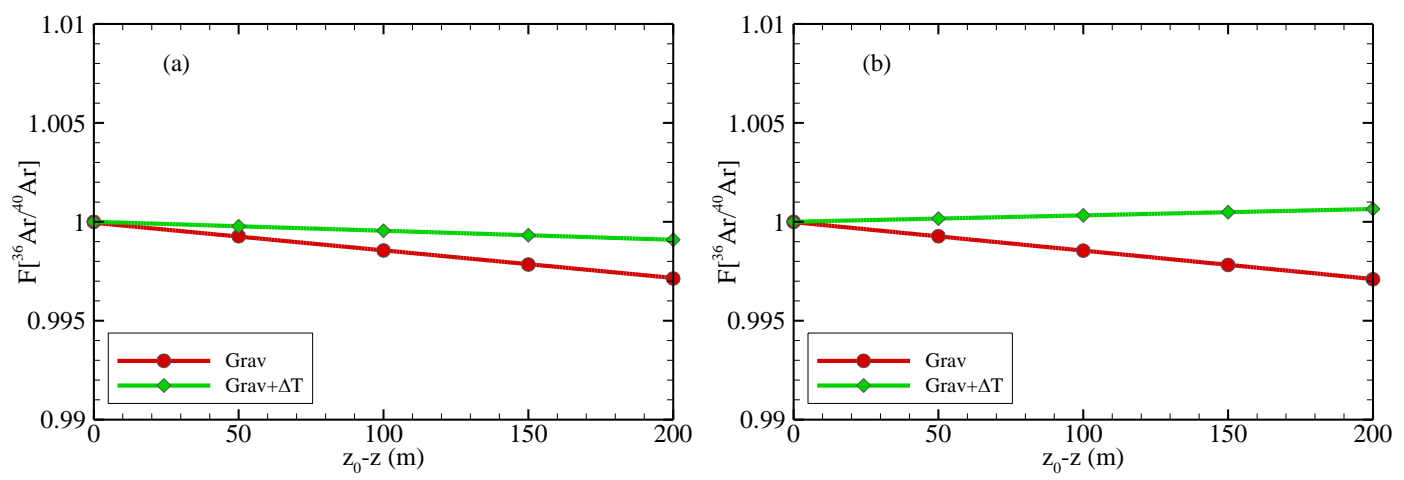